\title{
Development and evolution of methods for optimizing the modes of heating networks
}

\author{
Alexandr Lutsenko, ${ }^{1, *}$, and Nikolay Novitsky ${ }^{1}$ \\ ${ }^{1}$ Melentiev Energy Systems Institute of SB RAS, Pipeline Department, 664033 Irkutsk, Russia
}

\begin{abstract}
Both in Russia and abroad, energy efficiency problems are relevant, while heat supply systems have significant energy saving reserves, which can be realized by organizing optimal operating modes. In practice, the task of planning modes of heat supply systems is solved by multivariate mode calculations, while the choice of methods for organizing the modes is assigned to the specialist performing the calculations, which does not guarantee the optimality of the obtained modes. Automation of solving these problems is complicated by a number of factors. For these reasons, there are no methods and software systems suitable for wide practical application. This determines the relevance of developing separate methods and programs for calculating optimal modes of heat supply systems. The subject of this article is the tasks and methods of optimizing operation modes of heat supply systems using several objective functions at the same time. The object of application is hydraulically inextricable heat supply systems. It is assumed that the temperature schedule on heat source is set, the heat losses in the networks are eliminated, and their residual value can be neglected. In this case, the task is to optimize the hydraulic mode.
\end{abstract}

\section{Introduction}

Both in Russia and abroad, energy efficiency problems are relevant, while heat supply systems (HSS) have significant energy saving reserves [1], which can be realized by organizing optimal operating modes. In practice, the task of planning HSS modes is solved by multivariate mode calculations [2], while the choice of methods for organizing the modes is assigned to the specialist performing the calculations, which does not guarantee the optimality of the obtained modes. Automation of solving these problems is complicated by a number of factors: high dimension $\operatorname{HSS}[3,4]$, nonlinearity of the involved flow distribution models, the presence of several objective functions, etc. For these reasons, there are no methods and software systems suitable for wide practical application. This determines the relevance of developing separate methods and programs for calculating optimal HSS modes.

Recently, attention has been paid to optimizing HSS modes, but the bulk of research in this area is not applicable in the general case. Some works (for example, [5]) are devoted to HSS of small dimension. Many works $([6,7]$ etc.) use an approximation of the dependence of the objective function value on the mode parameters selected as a basis, which greatly complicates the correct accounting of discrete variables related to the composition of the operating equipment. In other cases, to overcome the dimension problem, aggregation of HSS schemes is used, which prevents taking into account the whole set of constraints and does not guarantee the required accuracy of solutions. A common approach is to use ready-made solvers. The main disadvantage of this approach is the impossibility of adapting methods to the specifics of tasks, which leads to too high computational costs. For example, in $[8,9]$ the physical model of the network was created in the Simulink / Matlab environment, the CPLEX solver is used to calculate the mode, and the ReMIND software is used for optimization. The use of genetic and evolutionary algorithms requires even greater computational costs. For example, in [10], a nested iterative cycle is used to minimize the cost of pumping the coolant. In the internal loop, the allowable mode is calculated using the SIMPLE algorithm. Using the genetic algorithm, the parameters determining the mode are changed on the external cycle. In a number of works, narrowly targeted objective functions are used, which does not allow applying the achieved results in the general case. For example, in [11] the total fuel consumption at heat sources (HS) is minimized. In [6, 7], the problem of optimal control of pumps at pumping stations (PS) was solved in order to minimize the consumed electric power. Basically, tasks related to operational management are considered, for example, [11 - 13]. Note that, there are practically no works on the problem of planning HSS modes of real dimension that occurs during the preparation for the heating season.

The subject of this article is the tasks and methods of optimizing HSS operation modes using several objective functions at the same time. The object of application is hydraulically inextricable HSS. It is assumed that the temperature schedule on HS are set, the heat losses in the networks are eliminated, and their residual value can be

Corresponding author: luc alex@mail.ru 
neglected. In this case, the requirements for providing consumers with thermal energy is reduced to the need to maintain their required coolant flows, and the task is to optimize the hydraulic mode (HM). The case of parallel operation of the same type of pumps on PS is considered as usual for HSS.

\section{Problem statement}

In order to optimize the HM HSS, it is necessary to find control actions that implement the mode that meets the admissibility requirements and reaches the set optimization goals. Energy saving requirements can be reduced to minimize the economic objective function. Minimizing the complexity of network preparation and reducing possible coolant leaks and the risks of emergency situations can be reduced to minimizing additional places for flow control and minimizing the total pressure in the network.

\subsection{The mathematical formulation of the problem.}

The main elements of HSS are pipeline sections, PS, consumers, HS, and ramification units. In the technological scheme (single-line representation, Fig. 1), pipeline sections and PS are represented by branches, and HS, consumers and ramification units are represented by nodes. In the design scheme (two-line representation, Fig. 2), the pipeline sections are depicted as two branches (supply and return pipelines), PS - as a branch with PS, consumers - as branches between the supply and return pipelines. HS can be modeled on the design scheme as a branch between the supply and return pipelines with a PS and a heating unit installed on it, the optimization of the operation mode of which represents a separate independent task [14]. We denote the set branches of the design scheme that model PS as $I_{P S}$, consumers - as $I_{C}$ and pipeline sections - as $I_{P L}$. Then $I_{P L} \cap I_{P S}=I_{P L} \cap I_{C}=I_{P S} \cap I_{C}=\varnothing \quad$ and $I_{P L} \cup I_{P S} \cup I_{C}=I-$ the set of all branches, $|I|=n$. We also introduce $J$ - the set of all nodes of the design scheme, $|J|=m$.

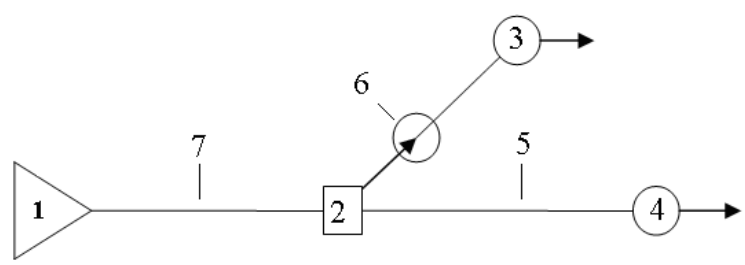

Fig. 1. An example of a technological scheme. 1 - HS; 2 ramification unit; 3.4 - consumers; 5, 7 - pipeline sections; 6 PS.

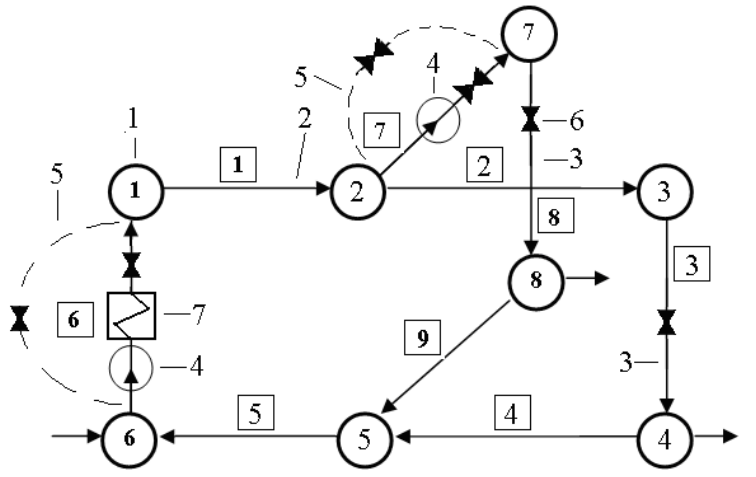

Fig. 2. An example of a design scheme. In circles, the numbers of nodes are indicated, in squares, the numbers of branches. 1 node $j, 2$ - branch $i, 3$ - consumer, 4 - PS, 5 - bypass line, 6 application point of the flow control means, 7 - heaters.

\subsubsection{Controlled flow distribution model.}

As the initial model, we will use the controlled flow distribution model [15], which consists of equations analogous to Kirchhoff's laws and equations describing the laws of the coolant flow over individual network elements:

$$
\mathbf{U}(\mathbf{R}, \mathbf{u})=\left(\begin{array}{c}
\mathbf{U}_{1}(\mathbf{x}, \mathbf{Q}) \\
\mathbf{U}_{\mathbf{2}}(\mathbf{P}, \mathbf{y}) \\
\mathbf{U}_{\mathbf{3}}(\mathbf{y}, \mathbf{x}, \mathbf{u})
\end{array}\right)=\left(\begin{array}{c}
A \mathbf{x}-\mathbf{Q} \\
\mathbf{A}^{T} \mathbf{P}-\mathbf{y} \\
\mathbf{y}-\mathbf{h}(\mathbf{x}, \mathbf{z}, \boldsymbol{\gamma}, \mathbf{\kappa})
\end{array}\right)=0 .
$$

The following notation is used here: $A$ - incidence $m \times n$-matrix of a connected oriented graph of the HSS design scheme; $\mathbf{Q}, \mathbf{P}-m$-dimensional vectors of nodal flow rates and pressures; $\mathbf{x}, \mathbf{y}-n$-dimensional vectors of flow rates and pressure drops on the branches; $\mathbf{h}(\mathbf{x}, \mathbf{z}, \gamma, \boldsymbol{\kappa})-n$-dimensional vector function with elements $h_{i}\left(x_{i}, z_{i}, \gamma_{i}, \kappa_{i}\right), \quad i=\overline{1, n}$ approximating the dependence of the pressure drop on the flow rate on the branch; $\gamma$ - vector of relative frequencies of rotation of the impellers of the pumps (or their diameters), we call the change in this parameter frequency regulation; к vector of the number of pumps turned on at the PS; $\mathbf{z}$ vector of relative hydraulic resistances (for example, increased by throttling). $\mathbf{R}=\{\mathbf{P}, \mathbf{Q}, \mathbf{x}, \mathbf{y}\}-$ set of variables corresponding to the mode parameters; $\mathbf{u}=\{\mathbf{z}, \boldsymbol{\gamma}, \boldsymbol{\kappa}\}-$ set of variables corresponding to control actions.

In the case of parallel operation of the pumps on the PS, as an approximation of the hydraulic characteristics of the $i$-th branch, we can take [15]:

$$
h_{i}\left(x_{i}, z_{i}, \gamma_{i}, \kappa_{i}\right)=z_{i} s_{i} x_{i}\left|x_{i}\right| / \kappa_{i}^{2}-\gamma_{i}^{2} H_{i} .
$$

The following notation is used here: $s_{i}-$ hydraulic resistance, $H_{i}$ - pressure increment created by pumps on PS. In (2), for modeling the absent or forbidden to change the control action, one can equate the corresponding variable constant. In addition, (2) can be considered as a generalization of the hydraulic characteristics of a controlled pipeline section if: $\kappa_{i}=1$, $H_{i}=0, \gamma_{i}=1$.

In case any mode parameter depends on the external environment, the corresponding variable is fixed. We 
call such parameters the boundary conditions. For HSS, such parameters are the pressure in the make-up nodes (nodes in which the coolant enters the HSS to compensate for the loss of coolant and withdrawals) and flow rates in all other nodes.

The requirements of the admissibility of the HM and the feasibility of control actions can be written as [15] $\underline{P}_{j} \leq P_{j} \leq \bar{P}_{j}, \quad \underline{y}_{i} \leq y_{i} \leq \bar{y}_{i}, \quad \underline{z}_{i} \leq z_{i} \leq \bar{z}_{i}, \quad \underline{\gamma}_{i} \leq \gamma_{i} \leq \bar{\gamma}_{i}$, $\kappa_{i} \gamma_{i} \underline{\chi}_{i} \leq x_{i} \leq \kappa_{i} \gamma_{i} \bar{\chi}_{i}, \quad \kappa_{i} \in \mathrm{K}_{i}, \quad i \in I, \quad j \in J$, where $\underline{P}_{j}, \bar{P}_{j}, \underline{y}_{i}, \bar{y}_{i}, \underline{\gamma}_{i}, \bar{\gamma}_{i}$ and $\underline{z}_{i}, \bar{z}_{i}$ - margins of change $P_{j}, y_{i}$, $\gamma_{i}$ and $z_{i} ; \mathrm{K}_{i}=\left\{0,1,2, \ldots, K_{i}\right\}, K_{i}$ - number of pumps installed on PS; $\underline{\chi}_{i}, \bar{\chi}_{i}$ - margins of change $x_{i}$ with $\gamma_{i}=1, \quad \kappa_{i}=1$. We introduce a vector of Boolean variables $\boldsymbol{\delta}$, whose components $\delta_{i}$ control the presence or absence of throttling on the $i$-th branch of the design scheme. We also replace the inequality $\underline{z}_{i} \leq z_{i} \leq \bar{z}_{i}$ by $\underline{z}_{i} \leq z_{i} \leq \underline{z}_{i}+\left(\bar{z}_{i}-\underline{z}_{i}\right) \delta_{i}$. Then the system of constraints takes the form

$$
\begin{aligned}
& \underline{P}_{j} \leq P_{j} \leq \bar{P}_{j}, \underline{y}_{i} \leq y_{i} \leq \bar{y}_{i}, \underline{z}_{i} \leq z_{i} \leq \underline{z}_{i}+\left(\bar{z}_{i}-\underline{z}_{i}\right) \delta_{i}, \\
& \underline{\gamma}_{i} \leq \gamma_{i} \leq \bar{\gamma}_{i}, \kappa_{i} \in \mathrm{K}_{i}, \kappa_{i} \gamma_{i} \underline{\chi}_{i} \leq x_{i} \leq \kappa_{i} \gamma_{i} \bar{\chi}_{i}, i \in I, j \in J
\end{aligned}
$$

\subsubsection{Objective functions.}

The variable component of the maintenance costs is $F_{C}=F_{C}^{E P}+F_{C}^{F}$, where $F_{C}^{E P}$ is the cost of electricity for pumping coolant to PS, $F_{C}^{F}$ is the fuel costs for heating the coolant. We denote $I_{H S} \subset I_{P S}$ as the set of HS. Then $F_{C}^{E P}=\sum_{i \in I_{P S}} c_{i}^{E P} N_{i}, F_{C}^{F}=\sum_{i \in I_{H S}} c_{i}^{C} B_{i}$, where $c_{i}^{E P}, N_{i}-$ the price of electricity and its consumption, $c_{i}{ }^{F}, B_{i}-$ the price of fuel and its consumption. For a given temperature schedule of HS, fuel consumption can be represented as a known function of the coolant flow rate. We approximate it by a square trinomial: $B_{i}\left(x_{i}\right)=\alpha_{0, i}+\alpha_{1, i} x_{i}+\alpha_{2, i} x_{i}^{2}$ [16]. The electrical power consumption on a separate PS is $N_{i}\left(x_{i}, \gamma_{i}, \kappa_{i}\right)=\beta_{0, i} \kappa_{i} \gamma_{i}^{3}+\beta_{1, i} \gamma_{i}^{2} x_{i}+\beta_{2, i} \gamma_{i} x_{i}^{2} / \kappa_{i}, \quad i \in I_{P S}$ [15]. Here $\beta_{0, i}, \beta_{1, i}, \beta_{2, i}$ - approximation coefficients of the dependence of the electric power consumption by an individual pump on the coolant flow rate, provided $\gamma=1$. Then the economic objective function will take the form $F_{C}(\mathbf{x}, \boldsymbol{\gamma}, \mathbf{\kappa})=\sum_{i \in I_{P S}} c_{i}^{E P}\left(\beta_{0, i} \kappa_{i} \gamma_{i}^{3}+\beta_{1, i} \gamma_{i}^{2} x_{i}+\beta_{2, i} \gamma_{i} x_{i}^{2} / \kappa_{i}\right)+$ $+\sum_{i \in I_{H S}} c_{i}^{C}\left(\alpha_{0, i}+\alpha_{1, i} x_{i}+\alpha_{2, i} x_{i}^{2}\right)$. The objective function of the number of control actions is $F_{z}(\boldsymbol{\delta})=\sum_{i \in I_{P L}} \delta_{i}$ [17], and the indicator of the general pressure level is $F_{P}(\mathbf{P})=\sum_{j \in J} P_{j} / m$ [17].

\subsubsection{Consideration of multiobjectiveness}

In preparation for the heating season, you need to find an HM that meets the requirements: 1) minimum maintenance costs; 2) the minimum complexity of adjustment activities; 3) the lowest level of pressure in HSS to minimize leaks, unproductive costs and risks of emergency situations. These requirements are listed in order of importance.

The following are known: topology of the design scheme; border conditions; coefficients of hydraulic and power characteristics $\left(\beta_{0, i}, \beta_{1, i}, \beta_{2, i}, s_{i}, H_{i}, i \in I\right)$; flow rate coefficients $\operatorname{HS}\left(\alpha_{0, i}, \alpha_{1, i}, \alpha_{2, i}\right)$;permissible limits of change of continuous variables; sets of possible values of integer variables $\left(\mathrm{K}_{i}, \delta_{i}, i \in I\right)$; the cost of electricity for each PS ( $\left.c_{i}^{E P}, i \in I_{P S}\right)$ and the cost of fuel for each HS $\left(c_{i}^{F}, i \in I_{H S}\right)$. It is required to determine the parameters of the optimal HM $(\mathbf{P}, \mathbf{Q}, \mathbf{x}, \mathbf{y})$ and the control actions necessary for its implementation $(\mathbf{z}, \gamma, \mathbf{\kappa}$, 反).

The specific mathematical formulation of the problem depends on the approach taken to account for multiobjective. As the main approach, it is proposed to use the vector optimization method in accordance with ordering of the requirements for $\mathrm{HM}$ by degree of importance.

Then the task is to find the parameters of the mode and the control actions that satisfy the following conditions: (1), (3), $F_{C}=F_{C}^{*}, F_{z}=F_{z}^{*}$ and $F_{P}=F_{P}^{*}$. Here: $\quad F_{C}^{*}=\min F_{C} \quad$ under constrains (1), (3); $F_{z}^{*}=\min F_{z}$ under constrains (1), (3) and $F_{C}=F_{C}^{*}$; $F_{P}^{*}=\min F_{P}$ under constrains (1), (3), $F_{C}=F_{C}^{*}$ and $F_{z}=F_{z}^{*}$. We write this problem as:

$$
\min _{\text {lex }}\left(F_{C}, F_{z}, F_{P}\right) \text { under constrains (1), (3). }
$$

\section{Hierarchical approach to optimizing HSS hydraulic modes}

To solve the problem of dimensionality of problem (4), the authors proposed a hierarchical approach to optimizing HM HSS [18], which allows us to separate the various types of discrete variables (integer and Boolean) and objective functions for various problems. This approach includes the following steps: 1) decomposition of HSS into main (MHN) and distribution (DHN) networks; 2) the search for the limits of permissible changes in the mode parameters at the decomposition point; 3) optimization of the MHN mode, taking into account the restrictions obtained in the previous step; 4) optimization of DHN modes taking into account the parameters of the MHN mode at the decomposition point.

The basic principles of HSS decomposition are as follows. MHN contains all HS, PS and multi-loop part of the network in a single-line representation. DHNs include tree-like passive networks to users. The decomposition point is the point where DHN joins the $\mathrm{MHN}$ in a single-line representation and two nodes in a bilinear representation, one of which is the connection 
point of the MHN and DHN supply pipelines, and the second is the return (Fig. 3). For MHN, the decomposition point is a generalized consumer with bilateral restrictions on the pressures in the supply and return piping and on the difference in these pressures. For DHN, the decomposition point is a generalized heat source.

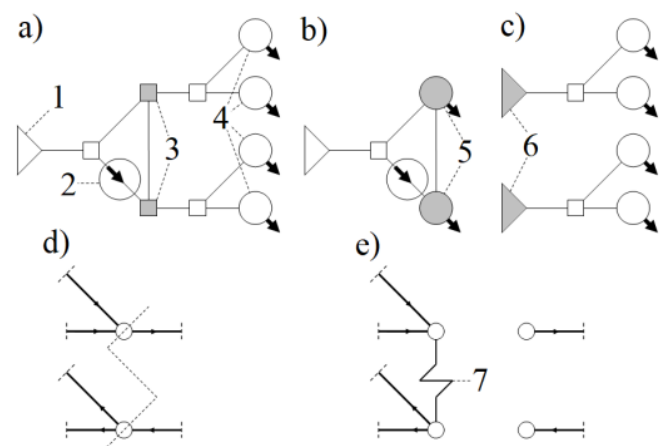

Fig. 3. Illustration of the principles of decomposition of the HSS. a) a single-line representation of an HSS; b) MHN; c) DHN; d) nodes of the division of hierarchical levels in a twoline representation; e) the result of decomposition on a fragment of a two-line representation. 1 - HS; 2 - PS; 3 - nodes of decomposition; 4 - consumers; 5 - generalized consumers; 6 - generalized HS; 7 - a branch with an equivalent characteristic replacing DHN.

Note that the economic objective function is related to the HM parameters and control actions that are relevant only to MHN. In practice, adjustment activities related to the installation of regulators, throttling washers, etc. occur only on DHN. This allows for the MHN to solve the following problem instead of problem (4):

$$
\min _{\text {lex }}\left(F_{C}, F_{P}\right) \text { under constrains (1), (3). }
$$

Due to the branched configuration, there is a fixed, easily computable flow distribution for DHN, as well as the ability to reduce the design scheme of DHN to one branch due to the equivalenting of serial and parallel branch connections. Therefore, instead of (1), we have:

$$
\mathbf{U}(\mathbf{R}, \mathbf{u})=\left(\begin{array}{c}
\mathbf{A}^{T} \mathbf{P}-\mathbf{y} \\
\mathbf{y}-\mathbf{h}(\mathbf{x}, \mathbf{z}, \boldsymbol{\gamma}, \boldsymbol{\kappa})
\end{array}\right)=0
$$

and problem (4) takes the form [19]:

$$
\min _{\text {lex }}\left(F_{z}, F_{P}\right) \text { under constrains (6), (3). }
$$

The study of the Pareto set of the following problem is also of practical importance:

$$
\min \left(F_{z}, F_{P}\right) \text { under constrains (6), (3). }
$$

The task of finding the limits of permissible changes in the mode parameters at the decomposition point is six optimization problems to be solved for each DHN: $\min P_{1}, \quad \max P_{1}, \quad \min P_{m}, \quad \max P_{m}, \min \left(P_{1}-P_{m}\right)$, $\max \left(P_{1}-P_{m}\right)$. Here $j=1$ is the connection node of the supply pipelines MHN and DHN, and $j=m$ is the return. All problems are solved under constrains (6) and (3).

\section{Optimization methods used in the hierarchical approach}

Given the approach used to take into account multicriteria, the optimization problem HM MHN (5) is reduced to the sequential solution of two problems:

$\min F_{C}$ under constrains (1), (3) and $\delta_{i}=0, i \in I_{P L}$

$\min F_{P}$ with (1), (3), $F_{C}=F_{C}^{*}$ and $\delta_{i}=0, i \in I_{P L}$

To solve problem (9), the authors developed a method [15], the essence of which is as follows. A triple nested iteration loop is used. The allowable HM is calculated on the internal cycle using the internal points method developed at ESI SB RAS [20, 21]. On the middle cycle, the value of the objective function is minimized by the bisection method. On the external loop, $\kappa_{i}$ values are searched by the continuous branch and bound method. Variables $\kappa_{i}$ are considered continuous on two internal iterative cycles, and on the external, their discreteness is achieved.

Problem (10) is solved similarly to problem (9) with the only difference being that an additional condition $F_{C}=F_{C}^{*}$ is imposed and $F_{P}$ is minimized.

To optimize HM DHN (task (7)), the authors developed a loop reducing dynamic programming method [19]. The essence of this method is as follows: 1) for each branch, all permissible pressure drop trajectories are constructed, while trajectories that have the same pressure drop $\left(y_{i}\right)$ but different pressures in the end nodes are considered different; 2) using special techniques of equivalent serial and parallel connection of branches, the design scheme and these trajectories are minimized (with rejecting both unacceptable and non-optimal pressure distribution options) to one branch with a single pressure drop trajectory corresponding to the optimal solution; 3) restoration of this solution to the original DHN scheme.

Potentially, this method can solve the problem of finding the limits of permissible changes in the mode parameters at the decomposition point in one application of this method for each DHN. At the moment, work is underway to adapt it to solve this problem. Previously, this problem was solved as 6 separate optimization problems for each DHN [18]. Each of these problems was solved using a double nested iterative loop. The allowable HM was calculated on the internal cycle using the internal point method, and on the external, the parameter under study was minimized or maximized.

\section{Investigation of the properties of the Pareto set of the optimization problem of the hydraulic mode of distribution heating networks}

We show that the Pareto set of problem (8) is discrete. We associate with each value of the vector $\boldsymbol{\delta}^{*}$ the value of $\tilde{F}_{P}\left(\boldsymbol{\delta}^{*}\right)$, equal to the minimum $F_{P}$, attainable at (6), (3) and $\boldsymbol{\delta}=\boldsymbol{\delta}^{*}$. If conditions (6), (3) and $\boldsymbol{\delta}=\boldsymbol{\delta}^{*}$ are incompatible, we set $\tilde{F}_{P}\left(\boldsymbol{\delta}^{*}\right)=\infty$. For all $\boldsymbol{\delta}$ giving the same value of $F_{z}$, we choose the best (smaller) value of $\hat{F}_{P}\left(F_{z}\right)=\min \left(\tilde{F}_{P}(\boldsymbol{\delta}) \mid \sum \delta_{i}=F_{z}\right)$. We discard all 
Table 1. PS pumping equipment's coefficients of characteristics

\begin{tabular}{|c|c|c|c|c|c|c|c|}
\hline PS & Number of pumps & $\mathrm{H}$ & $\mathrm{s}$ & $\mathrm{b}_{0}$ & $\mathrm{~b}_{1}$ & $\mathrm{~b}_{2}$ & $\mathrm{~b}_{3}$ \\
\hline 1 & 3 & 120 & 0,0000375 & 160 & 0,0706 & 0,000135 & $-0,0000001$ \\
\hline 2 & 5 & 80 & 0,00004 & 40 & 0,16 & 0 & 0 \\
\hline 3 & 3 & 120 & 0,000125 & 60 & 0,24 & 0 & 0 \\
\hline 4 & 2 & 60 & 0,0001 & 30 & 0,09 & 0 & 0 \\
\hline
\end{tabular}

$\hat{F}_{P}\left(F_{z}\right)$ satisfying at least one of the conditions: 1) $\left.\hat{F}_{P}\left(F_{z}\right)=\infty ; \quad 2\right) \quad \hat{F}_{P}\left(F_{z}\right) \geq \hat{F}_{P}\left(F_{z}-k\right), \quad k>0 . \quad$ The resulting set of points will be the Pareto set of problem (8). Obviously, it is a set of points on the plane $F_{P}-F_{z}$.

In [17], problem (7) was considered. To minimize $F_{z}$, the discrete branch-and-bound method was used, and among all variants with the same $F_{z}$ value, variants with a lower $F_{P}$ value are considered earlier than variants with a large $F_{P}$ value. Thus, the Pareto set of problem (8) is found. An example of such a set is presented in Fig. 4.

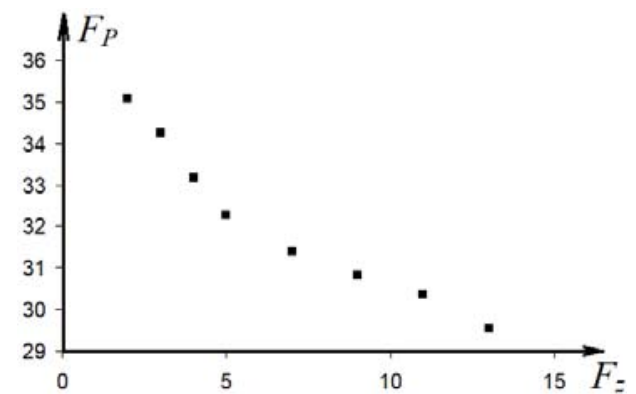

Fig. 4. An example of a Pareto set of optimization problems for DHN modes.

\section{Inverse Assignment Method}

The method of successive concessions [22] as applied to $(5)$ is as follows. For each objective function $\left(F_{C}, F_{z}, F_{P}\right)$ assignments $\left(\Delta F_{C}, \Delta F_{z}, \Delta F_{P}\right)$ are assigned, then the tasks are solved in series: 1) $F_{C}^{*}=\min F_{C}$ under constrains (1), (3); 2) $F_{z}^{*}=\min F_{z}$ under constrains (1), (3) and $F_{C} \leq F_{C}^{*}+\Delta F_{C} ; F_{P}^{*}=\min F_{P}$ under constrains (1), (3), $F_{C} \leq F_{C}^{*}+\Delta F_{C}$ and $F_{z} \leq F_{z}^{*}+\Delta F_{z}$.

In practice, sometimes the problem arises of minimizing the economic objective function with restrictions on $F_{z}$ :

$$
\min F_{C} \text { under constrains (1), (3) and } F_{z k} \leq F_{z k}^{*} \text {. }
$$

Here $k$ is the DHN number, $F_{z k}^{*}$ is a certain maximum number of control actions on the passive sections of the network for the $k$-th DHN, set by the specialist performing the calculations. Note that this problem is the inverse of the problems that arise when applying the method of successive concessions.

To solve this problem using a hierarchical approach to optimizing HM HSS, it is sufficient to require that the condition $F_{z} \leq F_{z k}^{*}$ be satisfied when searching for the limits of permissible changes in the mode parameters at the decomposition point for the $k$-th DHN.

\section{Example of hierarchical optimization of hydraulic mode}

The method described above was tested on the Baikalsk HSS calculation model (Fig. 5), in which part of the DHN was replaced by generalized consumers, the number of pumps in PS-2 was increased to five, and frequency regulation was allowed on all PSs. On the PS1 , the pumps are installed on the supply pipeline, for the rest of the PS - on the return. The number of pumps installed on the PS, their coefficients of characteristics are given in table 1 .

HSS has one HS, and all PS receive electricity at the same prices, so the electric power consumption by PS was the economic objective function. Optimization of HM HSS was carried out for the load case corresponding to the winter mode of operation of the HSS of Baikalsk. The calculations were carried out in accordance with the hierarchical approach to optimizing HM HSS.

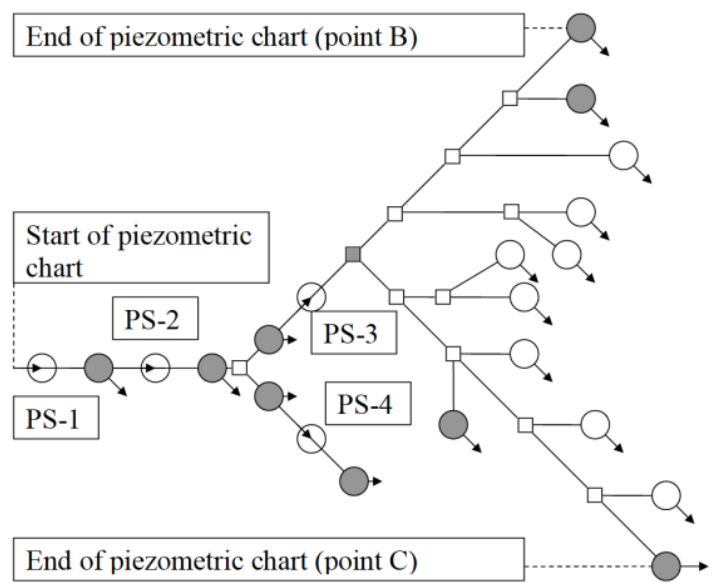

Fig. 5. Single-line representation of the HSS of Baikalsk

Table 2 shows the optimization results for HM MHN. It can be seen that the cost of pumping the coolant decreased by one and a half times. The number of controls on passive network sections after optimization was equal to one for the entire HSS considered.

Table 2. The optimization results for HM MHN

\begin{tabular}{|l|l|l|}
\hline $\begin{array}{l}\text { Calculation } \\
\text { option }\end{array}$ & $\begin{array}{l}\text { Power } \\
\text { Consumption } \\
(\mathrm{KW})\end{array}$ & $\begin{array}{l}\text { Average pressure } \\
\text { (m water) }\end{array}$ \\
\hline Feasible HM & 491,5 & 56 \\
\hline Cost reduction & 307,5 & 54,6 \\
\hline $\begin{array}{l}\text { Pressure } \\
\text { reduction }\end{array}$ & 307,5 & 43,49 \\
\hline
\end{tabular}


In fig. Figure 6 shows a piezometric graph of the optimal HM of a given HSS. Vertically shows the pressure in the pipeline, horizontally - the distance from HS. The vertical dashed line indicates the decomposition point. To the left of it is a piezometric graph of the MHS, to the right - DHS (the solid line for the $\mathrm{AB}$ route, the dotted line for the AC route). Rectangles indicate areas corresponding to PS (left) and throttling (right).

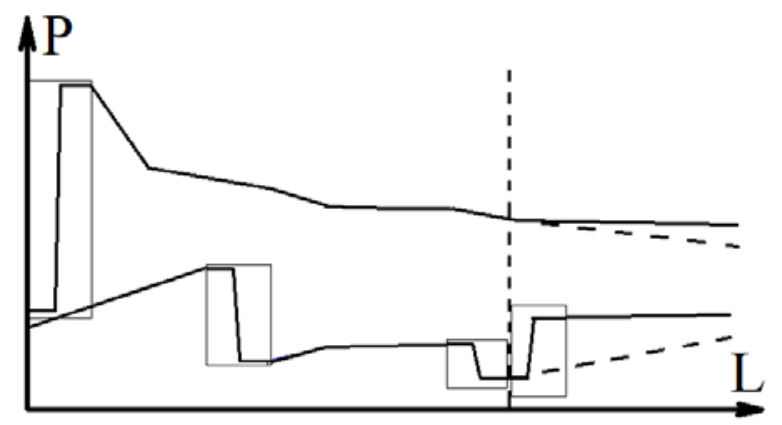

Fig. 6. The piezometric graph

\section{Findings}

The characteristic of the tasks of the organization of the HSS operating modes related to the development of commissioning measures in preparation for the next heating season is given. The optimization nature of these tasks is revealed, as well as the multi-purpose nature. A generalized mathematical formulation of the optimization problem for the hydraulic mode of the HSS is presented, which is a mixed, discrete-continuous optimization problem of large dimension with a vector objective function.

The characteristic developed by the authors of the hierarchical approach to optimizing the HSS modes is given, which provides both overcoming dimensional problems and significantly simplifying the general formulation for problems solved at different levels of the hierarchy.

A review of the developed methods for optimizing the MHN and DHN modes is presented, which have a rational combination of computational efficiency and guaranteed global solution, both with regard to taking into account the discreteness of part of the variables and the presence of several objective functions.

For the first time, an attempt is made to study the properties of the Pareto set of the formulated problem of optimizing DHN modes. A method for finding it is proposed. An original method of backward concessions is proposed within the framework of the developed hierarchical approach in the presence of a vector objective function.

A numerical example is given illustrating the performance and advantages of the proposed approach and methods for optimizing the hydraulic modes of the HSS.

The study was carried out within the framework of project III.17.4.3 of the fundamental research program of the SB RAS (AAAA-A17-117030310437-4)

\section{References}

1. V. Roshchanka, M. Evans, "Playing Hot and Cold: How Can Russian Heat Policy Find Its Way Toward Energy Efficiency?" National Technical Information Service, USA, 2010. 30p.

2. V. V. Tokarev, Z. I. Shalaginova, Thermal Engineering, 63 (2016)

3. S. Hodgson, District heating and CHP in Russia: Room for improvement, Cogeneration and On-site Power Production, (2009)

4. C. Tripodi, Energethica, (2012) (In Italian)

5. D. Buoro, P. Pinamonti, M. Reini. Appl. Energy, 124, (2014)

6. S. Cosentino, E. Guelpa, R. Melli, A. Sciacovelli, E. Sciubba, C. Toro, V. Verda. Proceedings of the ASME 2014 International Mechanical Engineering Congress and Exposition, (Montreal, Quebec, Canada, 2014)

7. A. Sciacovelli, E. Guelpa, V. Verda. ASME 2013 International Mechanical Engineering Congress and Exposition, 6A, (2013)

8. E. Guelpa, C. Toro, A. Sciacovelli, R. Melli, E. Sciubba, V. Verda, Energy, 102, (2016)

9. M. Vesterlund, J. Dahl. Energy Convers. Manag. 89 (2015)

10. A. Sciacovelli, E. Guelpa, V. Verda Proceedings of the ASME 2013 International Mechanical Engineering Congress and Exposition IMECE2013 (2013, San Diego, California, USA)

11. X.S. Jiang, Z.X. Jing, Y.Z. Li, Q.H. Wu, W.H. Tang, Energy, 64, (2013)

12. I.M. Mihkailenko Optimal control of district heating supply systems. (2003)

13. G.K. Voronovskiy Modifying on-line control practice for major heating systems under new economic conditions. (2002)

14. A.M. Claire, Effective methods of circuit-parametric optimization of complex heat power plants: development and application (2018)

15. N.N. Novitsky, A.V. Lutsenko, J. Global Optim. 66, (2016)

16. N.N. Novitsky, A.V. Lutsenko, Optimization of hydraulic modes of the main heating networks // Mathematical models and methods of analysis and optimal synthesis of developing pipeline and hydraulic systems. / Proceedings of the XIV AllRussian Scientific Seminar. (2014)

17. A.V. Lutsenko, N.N. Novitsky, Sci. Bull. NSTU., 64, (2016)

18. A.V. Lutsenko Hierarchical optimization of hydraulic modes of heat supply systems // System research in power engineering / Transactions of young scientists at MESI SB RAS, vol. 45, pp 2128,2015

19. A.V. Lutsenko, N.N. Novitsky Computational technologies. 23 (2018)

20. N.N. Novitsky, I.I. Dikin, Bulletin of the Russian Academy of Sciences. Energy, 5 (2003) 
21. A.V. Lutsenko, Study of problems and algorithmization of methods for calculating permissible hydraulic modes of heating networks // System research in power engineering / Transactions of young scientists at MESI SB RAS, vol. 42, pp 39-48, 2012

22. R. Steuer, Multiple Criteria Optimization: Theory, Computation and Application (1986) 